\title{
Bi-hierarchy Optimization of a Wind Farm Considering Environmental Impact
}

Tao, Siyu; Xu, Qingshan; Feijoo, Andres; Hou, Peng; Zheng, Gang

Published in:

IEEE Transactions on Sustainable Energy

Link to article, DOI:

$10.1109 /$ tste.2020.2964793

Publication date:

2020

Document Version

Peer reviewed version

Link back to DTU Orbit

Citation (APA):

Tao, S., Xu, Q., Feijoo, A., Hou, P., \& Zheng, G. (2020). Bi-hierarchy Optimization of a Wind Farm Considering Environmental Impact. IEEE Transactions on Sustainable Energy, 11(4), 2515 - 2524.

https://doi.org/10.1109/tste.2020.2964793

\section{General rights}

Copyright and moral rights for the publications made accessible in the public portal are retained by the authors and/or other copyright owners and it is a condition of accessing publications that users recognise and abide by the legal requirements associated with these rights.

- Users may download and print one copy of any publication from the public portal for the purpose of private study or research.

- You may not further distribute the material or use it for any profit-making activity or commercial gain

- You may freely distribute the URL identifying the publication in the public portal

If you believe that this document breaches copyright please contact us providing details, and we will remove access to the work immediately and investigate your claim. 


\title{
Bi-hierarchy Optimization of a Wind Farm Considering Environmental Impact
}

\author{
Siyu Tao, Student Member, IEEE, Qingshan Xu, Member, IEEE, Andrés Feijóo, Peng Hou, Member, \\ IEEE, and Gang Zheng
}

\begin{abstract}
The high penetration of wind energy in electrical power systems presents challenges for all operators. For the wind farm (WF) planners, one of these challenges is optimizing its layout with a set of constraints. This paper proposes a bi-hierarchy optimization scheme to determine the capacity and layout of a grid-connected WF. The environmental impacts involved by the installation of a WF have been taken into consideration in the problem. The first-layer model optimizes the WF capacity and configuration with minimized comprehensive generation cost of wind energy and two sets of constraints. The sound pressure level ( $S P L$ ) limit of the noise emitted by the wind turbines (WTs) is handled to be one of the constraints of the first-layer model. The second-layer model determines the generation schedule of other conventional generators. A Gaussian wake model is applied to calculate the effective wind speed for each WT. For the simulations, the WF is supposed to be integrated in the IEEE 30-bus test system. The wild goats algorithm (WGA) and the quadratic programming (QP) method are used to solve the problem. The simulation results validate the effectiveness of the proposed model and prove that environmental influences of WFs should not be ignored during the planning stage.
\end{abstract}

Index Terms - bi-hierarchy optimization, environmental effect, noise propagation, power flow, wake effect, wild goats algorithm, wind farm planning

$B_{m n}(S)$
$C_{E C, i}^{t}(€ / h r)$
$C_{g e n, i}^{t}(€ / h r)$
$C_{w f}(M V A)$
$C_{w f}^{o}(€ /$ day $)$
$C_{w f}^{l}(M V A)$
$C_{w f}^{u}(M V A)$
$C_{w i t}^{u n t}(M € / M W)$
$C_{w t}(€ /$ day $)$
$C_{e}(€ /$ day $)$
$C_{o}(€ /$ day $)$
$d(\%)$
$d_{r}(m)$
$D($ day $)$
$E_{w f}(M W h)$

\section{NOMENCLATURE}

Electrical susceptance of the line $m n$. Emissions penalty of the $i$-th generator at the $t$-th hour.

Generation cost of the $i$-th generator at the $t$-th hour.

WF capacity.

WF daily operation \& maintenance (O\&M) cost.

Lower bound of the WF capacity.

Upper bound of the WF capacity.

Unit cost of the WT.

WT daily equivalent investment.

Power grid daily environmental penalty cost.

Power grid daily operation cost.

Discount rate.

WT rotor diameter.

Total days in one year.

WF expected daily energy production.

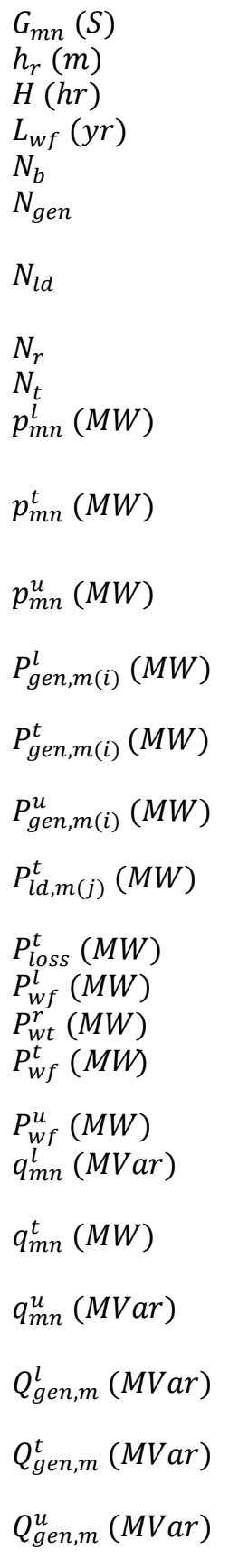

$G_{m n}(S)$

$L_{w f}(y r)$

$N_{b}$

$$
N_{l}
$$

\section{$N_{r}$}




$Q_{l d, m(j)}^{t}($ MVar $)$
$Q_{w f}^{t}(M V a r)$
$R_{s}(M W)$
$r_{r}(m)$
$S P L_{l i m}(d B(A))$
$U_{m(n)}^{l}(k V)$
$U_{m(n)}^{t}(k V)$
$U_{m(n)}^{u}(k V)$
$z_{0}(m)$
$z_{h}(m)$
$z_{r e f}(m)$
$\delta_{m}^{l}\left(^{\circ}\right)$
$\delta_{m n}^{t}\left({ }^{\circ}\right)$
$\delta_{m}^{u}\left(^{\circ}\right)$
$\lambda(€ / M W)$
$\rho\left(^{\circ} / M W\right)$
$\phi\left(^{\circ}\right)$

Reactive load value of the $m$-th ( $j$-th) bus at the $t$-th hour.

Reactive power output of the WF at the $t$ th hour.

Spinning reserve capacity.

WT rotor radius.

Upper limit of $S P L$.

Lower bound of the $m$-th ( $n$-th) bus voltage.

$m$-th ( $n$-th) bus voltage at the $t$-th hour.

Upper bound of the $m$-th ( $n$-th) bus voltage.

Surface roughness length.

WT hub height.

Wind speed measurement height.

Lower bound of voltage phase angle at the $m$-th bus.

Voltage phase angle difference between the $m$-th and the $n$-th buses at the $t$-th hour.

Upper bound of voltage phase angle at the $m$-th bus.

Network loss price.

Unit penalty price of pollutant emission. WF power factor angle.

\section{INTRODUCTION}

$\mathrm{W}$ IND power is regarded as an essential component of the modern energy supply and has been widely developed during the past decades. The worldwide cumulative wind power capacity reached 539 GW by the end of 2018 [1] and is anticipated to exceed $760 \mathrm{GW}$ by 2020 [2]. With the increasing penetration of wind power generation, wind energy plays a more and more essential role as an alternative of electrical generation in power systems. In view of this, the grid-connected WFs will have a huge influence on the electrical system operation. The load requirements and other generation sources' behavior will in turn have an impact on the determination of WF capacity and configuration. However, despite its general popularity, wind energy is facing boycotts in some countries due to potential environmental and health concerns that have caused increasing awareness for WF planners.

The optimization of the WF capacity and layout is a preliminary and important issue for WF designers and electrical network planners. Firstly, the active and reactive power output of a grid-connected WF will have a great impact on the power flow and will in turn influence the network losses and bus voltages. Secondly, the total power output of a WF is affected by the interaction among the WTs inside due to the phenomenon known as wake effect. This optimization problem is very complex and involves various economic costs and environmental effects. Many studies have been done in the WT optimal micro-siting problem since the pioneering works by Mosetti [3] and Grady [4]. In 2015, Long [5] proposed a twoechelon WF layout planning model in grid and Cartesian coordinates, solved by the random key genetic algorithm (RKGA) and the particle swarm optimization (PSO) algorithm. Hou put forward an offshore WF design method in 2015 [6] and a WF placement direction optimization and power dispatch strategy optimization in 2017 [7] respectively, both solved by means of the PSO algorithm. In 2016, Yang [8] studied the optimization of a WF and its application to power system reliability analysis, solved by the PSO algorithm. In 2018, Wang [9] investigated the utilization of the differential evolution (DE) algorithm in the WF layout optimization problem and designed a novel encoding mechanism to efficiently tackle this problem. A multi-objective optimization of a WF with a WT selection method was proposed in [10], solved by means of the harmony search (HS) algorithm and non-dominated sorting genetic algorithm-II (NSGA-II). Other milestone works of WF optimization include the research done by Sisbot [11], Kusiak [12], González [13], Chen [14], Shamshirband [15] and Sorkhabi [16].

The previous studies present two important deficiencies. Firstly, the WF capacity or the number of installed WTs is always decided beforehand. To our knowledge, the combined optimization of WF capacity and configuration in the gridconnected scenario has not been addressed before. However, in practice, the WF capacity determination and the WTs layout planning have mutual interaction and require to be jointly optimized to realize a cost-effective WF. Secondly, the objective function usually targets to find the trade-off between the minimization of the WF's own cost and the maximization of the power generation. The system operation costs and the environmental impacts brought by a WF have seldom been considered which usually embody in the following two aspects: 1) The network losses, the generation costs and the pollutant emissions in the power grid are reduced due to the presence of a WF. 2) WFs' operation would make noises that influence the neighboring's health, both for human beings and animals [16][17]. Therefore, both the positive and negative environmental impacts caused by a WF should not be ignored during the planning stage.

In order to overcome the drawbacks in the existent practices, a bi-hierarchy optimization method, which has been justified to be a suitable means to solve optimization problems with hierarchical features [18] is brought forward in this paper. The bi-hierarchy optimization has been successfully applied in reactive power optimization problems [19], in the plug-in electric vehicles coordinated dispatch problem [20], in the offshore WF collector system planning [21], and in the tidal current farm optimization problem [22][23], etc., and all these have shed light on the WF optimization problem.

This paper is organized as follows: in Section II, the power output of the WF is modeled; in Section III, the bi-hierarchy optimization model is proposed; Section IV introduces the optimization algorithm; Section V discusses the simulation results and the conclusions are exposed in Section VI.

\section{Modeling OF A WF}

\section{A. WT Power Curve}

Compared with a piecewise polynomial function, the Logistic function [24] expressed in (1) is non-piecewise and is closer to the real WT power curve. It is used to represent the WT power curve in this study.

$$
P_{w t}(v)=P_{w t}^{r}\left(1+\exp \left(\frac{2 s\left(v_{i p}-v\right)}{P_{r}-P_{i p}}\right)\right)^{-1}
$$


where $s=\left.\frac{d P}{d v}\right|_{v=v_{\text {ip }}}, P_{i p}$ and $v_{i p}$ are the power and wind speed at the inflection point of the WT power curve.

\section{B. Wake Model}

The Gaussian wake model (Fig. 1) which has been justified can better describe the real wake, states that [25]:

$$
\left\{\begin{array}{c}
v_{x, r}=\left(1-K(x) \exp \left(-\frac{r^{2}}{2 \sigma^{2}}\right)\right) v_{0} \\
K(x)=1-\sqrt{1-\frac{C_{T}}{2\left(\frac{\sigma}{r_{r}}\right)^{2}}} \\
\sigma=\frac{r_{x}}{2}=\frac{r_{r}}{2}+\frac{\alpha}{2} x
\end{array}\right.
$$

where $v_{x, r}$ is the wind speed at distance $x$ and radius $r, \sigma$ denotes the characteristic width of the wake, $K(x)$ is an undetermined coefficient of $x, C_{T}$ is the thrust coefficient and $\alpha$ is the entrainment constant which can be obtained from (3).

$$
\alpha=\frac{0.56}{\ln \frac{z_{h}}{z_{0}}}
$$

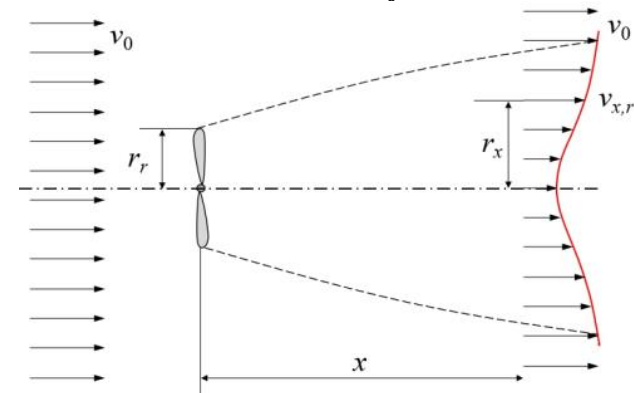

Fig. 1. Gaussian wake model [25].

In this model, the radius of the wake expands indefinitely due to the characteristic of the exponential function. Consequently, all WTs located downstream with respect to a given one are in the wake regardless of the distance between them and no WT will be partially in the wake of another WT [26].

\section{WF Output Power Calculation}

A rotation of coordinates is operated in advance to ensure the wind is always in front of the WTs. By using the method proposed in [26], for a given inflow wind velocity $\boldsymbol{v}=\left(v_{0}, \theta\right)$ with wind speed $v_{0}(\mathrm{~m} / \mathrm{s})$ and wind direction $\theta\left({ }^{\circ}\right)$, the WTs' coordinates should be transformed from the original layout $[X, Y]$ to the new one $\left[X^{\prime}, Y^{\prime}\right]$ according to (4).

$$
\left[X^{\prime}, Y^{\prime}\right]=[X, Y]\left[\begin{array}{cc}
\cos \theta & \sin \theta \\
-\sin \theta & \cos \theta
\end{array}\right]
$$

When the relative distances between WTs in the new coordinates are determined, the wind speed of the $i$-th WT $(i=$ $\left.1,2, \ldots, N_{t}\right), v_{i}$ can be calculated by (5) [26].

$$
v_{i}=v_{0}-\sqrt{\sum_{j=1}^{N_{t}} t_{i j} \Delta v_{i j}^{2}}
$$

where $t_{i j}$ is a binary variable that equals 1 when WT $i$ is in the wake of WT $j$ and 0 otherwise. Because in the Gaussian model, wake expands indefinitely, $t_{i j}$ assumes the value of 1 provided that WT $j$ is located in front of WT $i$ for a given wind direction $\theta$. Therefore, the velocity deficit only relies on the location of each WT inside the WF and the direction of the wind. $\Delta v_{i j}$ is the wake deficit of the $i$-th WT caused by the $j$-th WT.
The power output of the $i$-th WT $P_{w t}\left(v_{i}\right)$ can be calculated according to (1). In this way, the total power output of the WF $P_{w f}$ can be obtained according to (6).

$$
P_{w f}=\sum_{i=1}^{N_{t}} P_{w t}\left(v_{i}\right)
$$

A WF can be regarded as a PQ load bus [27][28] when integrated in a network. The WF total injected active power $P_{w f}$ and reactive power $Q_{w f}$ are calculated according to (6) and (7) respectively.

$$
Q_{w f}=P_{w f} \tan \phi
$$

where $\phi$ is the power factor angle of the WF.

\section{BI-HIERARCHY OPTIMIZATION MODEL}

When tackling complex optimization problems with multilevel variables, the bi-hierarchy method has two advantages over the uni-hierarchy optimization. Firstly, it remarkably degrades the computational scale and reduces the complexity of the developed model. Besides, it significantly relieves the loading on the communication network [18]. Fig. 2 shows the general framework of the WF bi-hierarchy optimization.

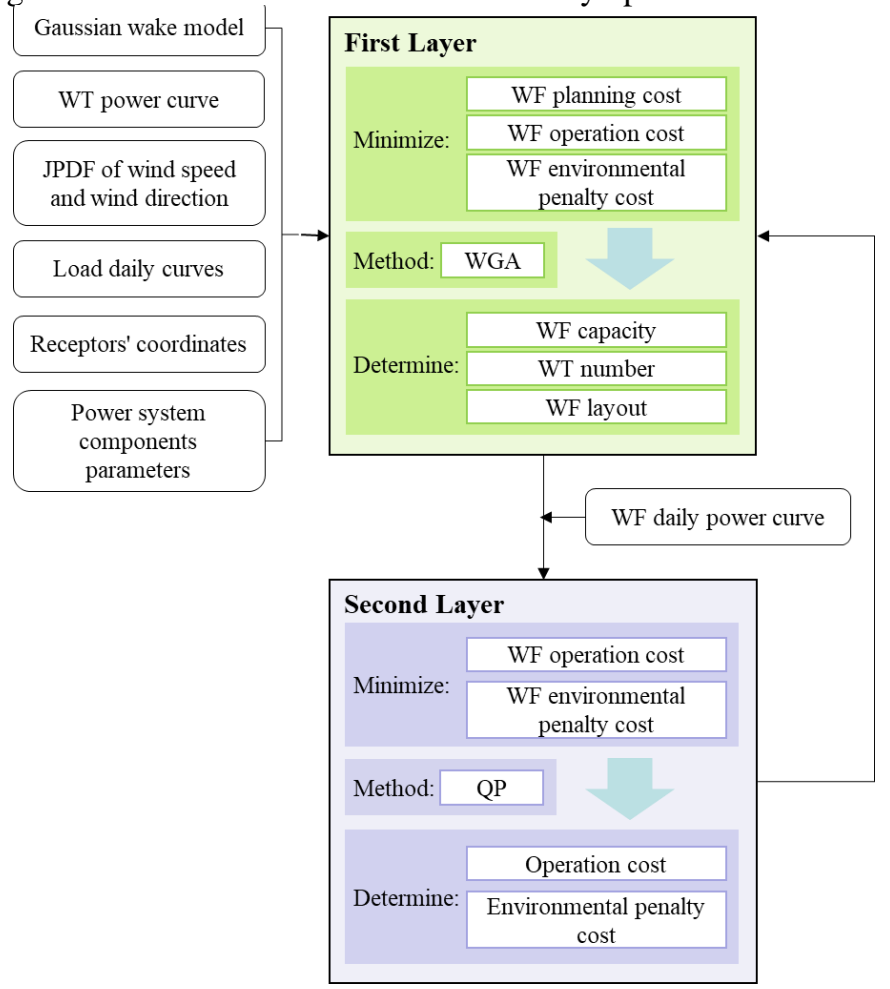

Fig. 2. Bi-hierarchy optimization framework.

The interaction mechanism between the first and the second layer models is as follows. The overall optimization of WF sizing and WT micro-siting is formulated by the first layer with the objective to minimize the comprehensive generation cost of wind power and decide the optimal WF capacity, WT number and layout. The second layer is nested in the first layer via procuring the optimal generation schedule of other generators in the electrical network and producing the minimum operation and environmental penalty costs for the first layer model. The generation schedule acquired from the second layer model is affected by the outcome of the first layer model including the scheme of WTs integration and placement. Also, the optimized results of the first layer model are related to the generation and 
the environmental penalty costs obtained from the second layer model.

\section{A. The First Layer Model}

In order to optimize the WF capacity and layout with minimized comprehensive generation cost of wind energy, the first-layer problem is formulated as (8).

$$
\begin{aligned}
& \min F_{1}(\boldsymbol{T})=\frac{C_{w t}+C_{o}+C_{e}}{E_{w f}} \\
& \text { s.t. }\left\{\begin{array}{c}
W B_{x}^{l} \leq T_{x}^{i} \leq W B_{x}^{u} \\
\text { cstr. } 1\left\{\begin{array}{c}
W B_{y}^{l} \leq T_{y}^{i} \leq W B_{y}^{u} \\
\sqrt{\left(T_{x}^{i}-T_{x}^{j}\right)^{2}+\left(T_{y}^{i}-T_{y}^{j}\right)^{2}} \geq 6 d_{r} \\
C_{w f}^{l} \leq C_{w f} \leq C_{w f}^{u} \\
S P L\left(\boldsymbol{T}, \boldsymbol{R}^{k}\right) \leq S P L_{l i m}
\end{array}\right. \\
i, j \in\left\{1,2, \ldots, N_{t}\right\}, i \neq j, k \in\left\{1,2, \ldots, N_{r}\right\} \\
N_{t} \in\left\{N_{t}^{\min }, N_{t}^{\min }+1, \ldots, N_{t}^{\max }\right\}
\end{array}\right.
\end{aligned}
$$

where $\boldsymbol{T}=\left(T_{x}^{i}, T_{y}^{i}\right), i=1,2, \ldots, N_{t}$ represents the matrix of the WTs' coordinates, $\boldsymbol{R}^{k}=\left(R_{x}^{k}, R_{y}^{k}\right), k=1,2, \ldots, N_{r}$ denotes the matrix of the noise receptors' coordinates. $F_{1}$ is the generation cost of unit wind energy consisting of the daily equivalent investment $C_{w t}$, the operation costs $C_{o}$ of the power grid and its environmental penalty $\operatorname{cost} C_{e}$. The minimum and maximum numbers of WTs are calculated by (9).

$$
N_{t}^{\min (\max )}=\operatorname{round}\left(\frac{P_{w f}^{l(u)}}{P_{w t}^{r}}\right)
$$

where round is the rounding function.

The constraints in the first-layer model are constituted in two parts.

Cstr. 1 concerns: 1) the boundaries of the WF planning area, i.e. $W B_{x}^{l}, W B_{x}^{u}, W B_{y}^{l}$ and $W B_{y}^{u}$; 2) the minimum allowable distance between WTs; 3 ) the lower and upper bounds of the WF capacity, i.e. $C_{w f}^{l}$ and $C_{w f}^{u}$; 4) the $S P L$ limit of the WF at each of the $N_{r}$ receptors, which is constrained to be lower than $40 d B(A)$. This value was recommended by the World Health Organization (WHO) [29].

$\operatorname{SPL}\left(\boldsymbol{T}, \boldsymbol{R}^{k}\right)$ is the equivalent continuous downwind octaveband $S P L$ of the whole WF and it can be calculated according to $(10)$.

$$
\operatorname{SPL}\left(\boldsymbol{T}, \boldsymbol{R}^{k}\right)=10 \log \left(\sum_{i=1}^{N_{t}} \sum_{j=1}^{8} 10^{0.1\left(L_{f}^{(i, j)}(\boldsymbol{T}, \boldsymbol{R})+A_{f}^{(j)}\right)}\right)
$$

where $L_{f}^{(i, j)}$ is the equivalent continuous downwind octaveband $S P L$ at each receptor location computed for each sound source at each of the eight octave bands $j$ with nominal midband frequencies $(63 \mathrm{~Hz}-8 \mathrm{kHz})$ [30]. $A_{f}^{(j)}$ is the standard Aweighting coefficients [16].

$S P L$ for each octave-band $\left(L_{f}\right)$ can be written as in (11).

$$
L_{f}=L_{w}+D_{c}-A_{w}(f)
$$

where $L_{w}$ is the octave-band sound power emitted by the noise source, $D_{c}$ is the directivity correction for sources that are not omni-directional ( $D_{c}$ is set to be 0 in this study), $A_{w}(f)$ is the octave-band attenuation. The detailed calculation procedures are available in [30].

An example from the Horns Rev I WF of the relationship between $S P L$ and the distance from the sound source is shown in Fig. 3. It reveals that the WTs, when deemed as noise sources, should be located as far as possible from the receptors.

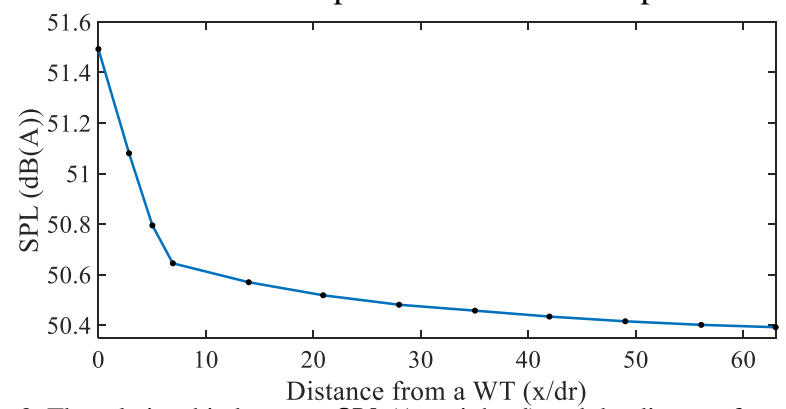

Fig. 3. The relationship between SPL (A-weighted) and the distance from the sound source [17].

Cstr. 2 is the set of power flow constraints at the $t$-th hour including: 1) active and reactive power balances; 2) bus voltage magnitude and phase angle constraints; 3) regular generators' active and reactive power output constraints; 4) bus active and reactive power flow constraints.

$C_{w t}$ stands for the WTs daily equivalent investment cost and can be derived by using (12).

$$
C_{w t}=N_{t} C_{w t}^{u n i t} P_{w t}^{r} \frac{d(1+d)^{L} w f}{\left((1+d)^{\left.L_{w f}-1\right) D}\right.}
$$

$C_{o}$ is the total operation cost including the WF daily O\&M cost, generation cost of other generators in the power grid and network loss cost. It can be calculated by (13).

$$
C_{o}=C_{w f}^{o}+C_{g e n}^{o}+C_{\text {loss }}^{o}
$$

where $C_{w f}^{o}$ is the WF daily O\&M cost and can be calculated by (14).

$$
C_{w f}^{o}=\frac{O P E X_{u n i t} \cdot C_{w f}}{D}
$$

where $O P E X_{\text {unit }}(€ / M W / y r)$ is the WF unit annual O\&M cost.

$C_{g e n}^{o}$ can be obtained from the second-layer model.

$C_{\text {loss }}^{o}$ is the network loss cost which is calculated by (15).

$$
C_{\text {loss }}^{o}=\sum_{t=1}^{H} P_{\text {loss }}^{t} \lambda
$$

where $P_{\text {loss }}^{t}$ is the network loss at the $t$-th hour which can be computed by (16).

$$
P_{\text {loss }}^{t}=\sum_{i=1}^{N_{g e n}} P_{g e n, i}^{t}+P_{w f}^{t}-\sum_{j=1}^{N_{l d}} P_{l d, j}^{t}
$$

where $P_{g e n, i}^{t}$ can be obtained from the second-layer model. $P_{w f}^{t}$ can be acquired from the typical daily curves of wind speed and wind direction.

$C_{e}$ is the pollutant emission penalty cost and can be formulated by (17).

$$
C_{e}=\sum_{t=1}^{H} \sum_{i=1}^{N_{g e n}} C_{E C, i}^{t}
$$

$C_{o}$ and $C_{e}$ are both determined by the second-layer model and sensitive to the power output of the WF. The first and the second-layer models interact with each other by $C_{o}$ and $C_{e}$.

$E_{w f}$ is the expected daily energy production of the WF and can be estimated by (18).

$$
E_{w f}=H \cdot \sum_{i=1}^{N_{t}} \int_{0^{\circ}}^{360^{\circ}} \int_{v_{\text {in }}}^{v_{\text {out }}} P_{w t}\left(v_{i}\right) \cdot J P D F(v, \theta) d v d \theta
$$
where $\operatorname{JPDF}(v, \theta)$ stands for the joint probability distribution function of wind speed and wind direction. $v_{\text {in }}$ and $v_{\text {out }}$ are the cut in and cut out wind speeds of the WT. 


\section{B. The Second Layer Model}

The aim of the second layer model is to plan the generation schedule of other generators in the power grid. It is formulated as an optimal economic dispatching model given as (19).

$$
\begin{gathered}
\min F_{2}\left(\boldsymbol{P}_{\text {gen }}\right)=\sum_{t=1}^{H} \sum_{i=1}^{N_{g e n}} C_{g e n, i}^{t}+C_{E C, i}^{t} \\
\text { s.t. }\left\{\begin{array}{c}
P_{w f}^{t}+\sum_{i=1}^{N_{g e n}} P_{g e n, i}^{t}-\sum_{j=1}^{N_{l d}} P_{l d, j}^{t}=0 \\
P_{w f}^{t}+\sum_{i=1}^{N_{g e n}} P_{g e n, i}^{u}-\sum_{j=1}^{N_{l d}} P_{l d, j}^{t}-R_{s}>0 \\
P_{g e n, i}^{l} \leq P_{g e n, i}^{t} \leq P_{g e n, i}^{u}
\end{array}\right.
\end{gathered}
$$

$C_{g e n, i}^{t}$ can be expressed as a quadratic function such as in (20) [31].

$$
C_{g e n, i}^{t}\left(P_{g e n, i}^{t}\right)=a_{o, i}+a_{1, i} P_{g e n, i}^{t}+a_{2, i}\left(P_{g e n, i}^{t}\right)^{2}
$$

where $a_{o, i}, a_{1, i}, a_{2, i}(€ / M W)$ are the cost coefficients of the $i$ th generator, determined according to the generators' attributes.

$C_{E C, i}^{t}$ is the penalty cost of pollutant emission at the $t$-th hour and can be expressed by (21).

$$
C_{E C, i}^{t}\left(P_{\text {gen }, i}^{t}\right)=\text { Pollut }_{i}^{t}\left(P_{\text {gen }, i}^{t}\right) \cdot \rho_{i}
$$

where $\rho_{i}(€ / M W)$ is the unit penalty price which can be derived according to the method in [31]. Pollut ${ }_{i}^{t}$ is the amount of pollutant released by the $i$-th generator at the $t$-th hour, and can be evaluated by (22) [31].

$$
\operatorname{Pollut}_{i}^{t}\left(P_{\text {gen }, i}^{t}\right)=b_{o, i}+b_{1, i} P_{\text {gen }, i}^{t}+b_{2, i}\left(P_{\text {gen }, i}^{t}\right)^{2}
$$

where $b_{o, i}, b_{1, i}$ and $b_{2, i}(l b / M W)$ are the emission coefficients of the $i$-th generator.

The three constraints represent the power balance of the power grid, the spinning reserve capacity restriction and the generators' output power limitation. The optimal generation schedule can be decided by the second-layer model, and the components $C_{o}$ and $C_{e}$ in $F_{1}$ of the first-layer model can be accordingly obtained from the second-layer model.

\section{OPTIMIZATION AlgORITHM}

\section{A. The WGA}

The WGA is a newly-developed evolutionary algorithm (EA) inspired by the climbing behavior of wild goats [32]. It possesses the features of mutation, swarm intelligence, memory-based and search in groups. The WGA is used to solve the first-layer model.

In the WGA, the initial values assigned to the variables are $w g \mathrm{~s}$, which denote wild goats. Each $w g$ is a solution of the optimization problem.

$$
w g_{i}=\left[\boldsymbol{x}_{\boldsymbol{i}}, \boldsymbol{y}_{\boldsymbol{i}}\right], i=1,2, \ldots, N_{w g}
$$

where $N_{w g}$ is the wild goats total number.

The evaluated value of each $w g$ can be expressed as in (24).

$$
F_{1}\left(w g_{i}\right)=F_{1}\left(\boldsymbol{x}_{\boldsymbol{i}}, \boldsymbol{y}_{\boldsymbol{i}}\right), i=1,2, \ldots, N_{w g}
$$

The weight $W_{i}$ of each $w g$ can be calculated by (25).

$$
W_{i}=\exp \left(-2 \frac{F_{1}\left(w g_{i}\right)-\min _{j}\left\{F_{1}\left(w g_{j}\right)\right\}}{\sum_{j=1}^{N_{w g}}\left(F_{1}\left(w g_{j}\right)-\min _{j}\left\{F_{1}\left(w g_{j}\right)\right\}\right)}\right)
$$

The $w g$ s with the highest value of $W$ are selected as leaders of groups $G$.

$$
\left\{\begin{array}{c}
G_{1}=\left\{w g_{1}, \ldots, w g_{N_{G_{1}}}\right\} \\
\vdots \\
G_{N_{g}}=\left\{w g_{N_{G_{N_{g}-1}}+1}, \ldots, w g_{N_{f}}\right\}
\end{array}\right.
$$

where $N_{g}$ is the number of groups at the start of the algorithm, and $N_{f}=N_{w g}-N_{g}$.

Then after the procedures of: 1) movement within groups; 2) reevaluation; 3) groups' cooperation and mutation of young goats; 4) movement to form one group, the optimum solution is obtained until reaching the maximum termination $I t_{\max }$.

In procedure 1), the movement of $w g \mathrm{~s}$ uses a vector $v$ similar to the one used by the PSO algorithm.

The leaders' movement is determined by (27).

$v(t+1)=w \cdot v(t)+R \cdot \mathrm{rand} \cdot(p(t)-w g(t))+c(t)(27)$ where $w$ and $R$ are the inertia weight and the personal learning coefficient respectively. $p$ is the best attempt and $c$ is an auxiliary parameter. rand is a random number, rand $\in[0,1]$.

The followers' movement is determined by (28).

$$
\begin{array}{r}
v(t+1)=w \cdot v(t)+R \cdot \operatorname{rand} \cdot(p(t)-w g(t))+ \\
W_{l_{k}}(t) \cdot \operatorname{rand} \cdot\left(w g_{l_{k}}(t)-w g(t)\right)+c(t)
\end{array}
$$

where $k$ indicates the index of groups.

The update of each $w g$ 's position is given by (29).

$$
w g_{i}(t+1)=w g_{i}(t)+v_{i}(t+1), \quad i=1, \cdots, N_{w g} \text { (29) }
$$

In procedure 3 ), the cooperation of $w g \mathrm{~s}$ is given by (30).

$$
G_{i}=G_{i}+\left\{w g_{k} \mid k \in j, \min _{w g \in j}\{W\}=W_{k}\right\}
$$

The mutation of $w g \mathrm{~s}$ uses an operator similar to that of the genetic algorithm (GA).

$$
w g_{i}(t+1)=w g_{i}(t)+\operatorname{rand} \cdot\left(\operatorname{rand}-w g_{i}(t)\right)
$$

The detailed description of the WGA can be found in [32].

\section{B. The QP algorithm}

The QP [33] is a gradient-based optimization algorithm using differentiating of the objective function and an iterative procedure which can only be used to solve the continuously differentiable function. QP is used to solve the second-layer optimization problem. Afterwards, $C_{o}$ and $C_{e}$ are acquired and sent to the first-layer model.

\section{Solution Procedures of the Optimization Problem}

The first and second-layer models are linked by the WF installed capacity and layout and generation schedule of other generators in the power grid. This can be better understood with the help of the flow chart in Fig. 4 of this bi-hierarchy optimization problem. 


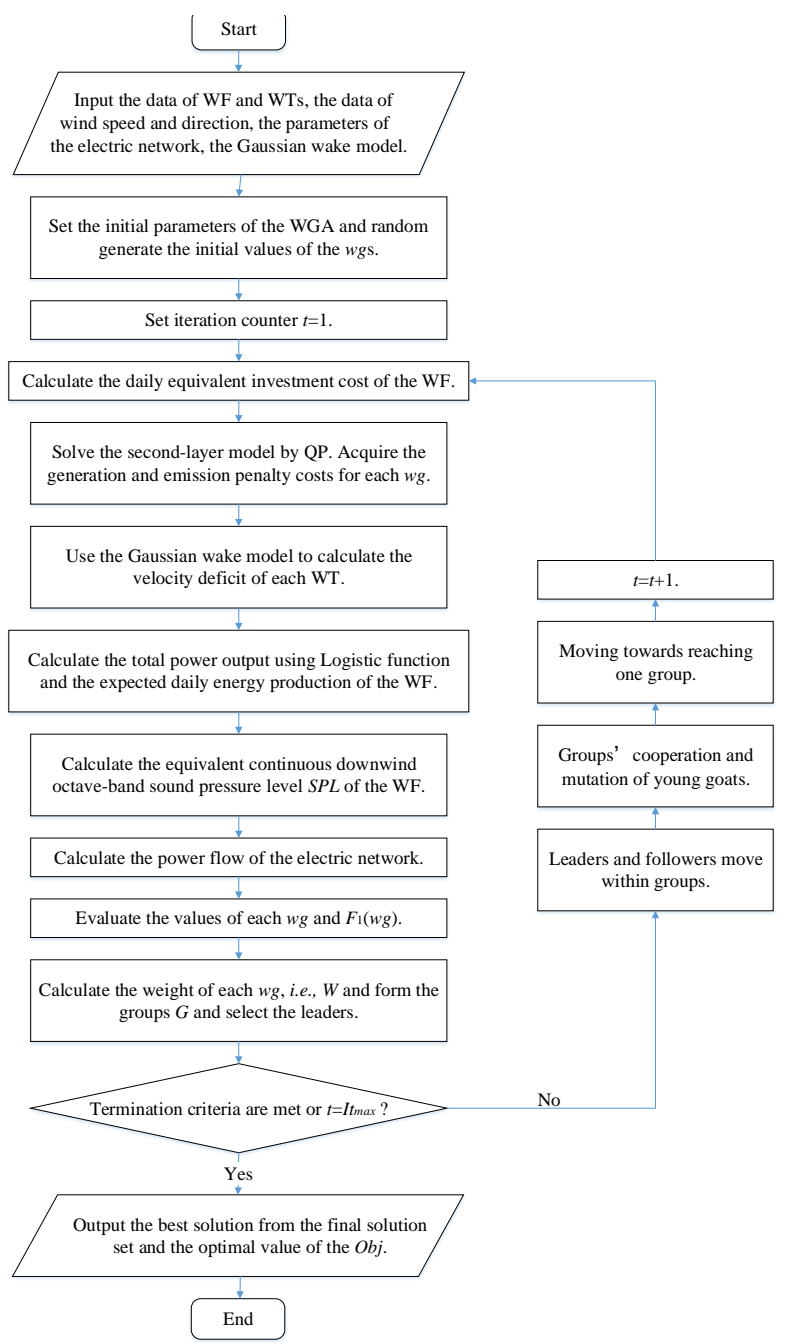

Fig. 4. Flow chart of the bi-hierarchy optimization.

\section{CASE Study}

\section{A. Test System and Data Source}

The WF to be optimized occupies a rectangle area and its basic information is given in Table I. The planning area of the WF is given in Fig. 5 where the red dots on the boundaries denote the noise receptors. In the WF layout planning stage, all the dwellings in the neighborhood are regarded to be potential noise receptors. Receptors No.1-8, marked in blue circles are in the eight typical directions (NW, N, NE, E, SE, S, SW, W).

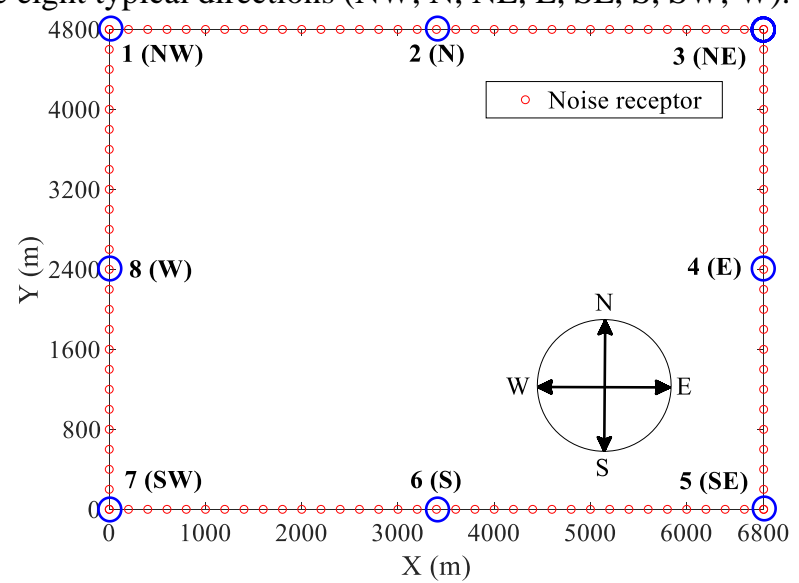

Fig. 5. Planning area of the WF.
TABLE I

INFORMATION OF THE WF

\begin{tabular}{cccccc}
\hline \hline Length $(\mathrm{m})$ & Width $(\mathrm{m})$ & $L_{w f}(y r)$ & $z_{0}(\mathrm{~m})$ & $z_{\text {ref }}(\mathrm{m})$ & $\cos \phi$ \\
\hline 6800 & 4800 & 25 & 0.0001 & 62 & 0.9 \\
$C_{w f}^{l}(M V A)$ & $C_{w f}^{u}(M V A)$ & $\begin{array}{c}\text { OPEX } \\
(€ / M W / y r\end{array}$ & $d(\%)$ & $N_{r}$ & $h_{r}(m)$ \\
120 & 180 & 106 & 5.39 & 116 & 1.5 \\
\hline \hline
\end{tabular}

The WTs installed in the WF are of the type Vestas V80-2 $M W$, which were installed in Horns Rev I offshore WF. The parameters of V80 are given in Table II. The power output [34] and sound power emittance $\left(L_{w}\right)$ [16] curves of this WT are given in Fig. 6.
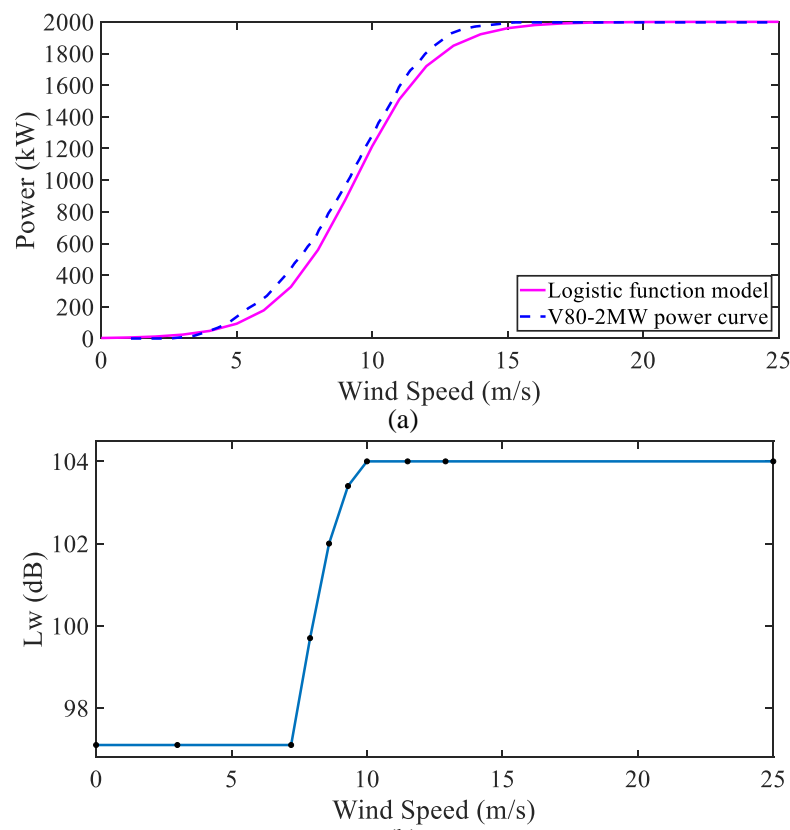

(b)

Fig. 6. Relationship of (a) power output and (b) sound power emittance of V80-2MW at different wind speeds.

TABLE II

PARAMETERS OF VESTAS V80

\begin{tabular}{ccccc}
\hline \hline$d_{r}(m)$ & $P_{w t}^{r}(k W)$ & $v_{\text {in }}(\mathrm{m} / \mathrm{s})$ & $v_{r}(\mathrm{~m} / \mathrm{s})$ & $v_{\text {out }}(\mathrm{m} / \mathrm{s})$ \\
\hline 80 & 2000 & 4 & 15 & 25 \\
$z_{h}(\mathrm{~m})$ & $C_{T}$ & $P_{\text {ip }}(\mathrm{kW})$ & $v_{\text {ip }}(\mathrm{m} / \mathrm{s})$ & $\mathrm{s}(\mathrm{kW} \cdot \mathrm{s} / \mathrm{m})$ \\
100 & 0.2 & 1091.2 & 9.37 & 313.7514 \\
\hline \hline
\end{tabular}

The WF is supposed to be integrated in the IEEE 30-bus test system. The parameters of the generators in this system are given in Table III [22]. An exchange rate is used for the conversion of dollars to euros.

TABLE III

PARAMETERS OF THE GENERATORS

\begin{tabular}{ccccccccc}
\hline $\begin{array}{c}\text { Bus } \\
\text { No. }\end{array}$ & $\begin{array}{c}P_{\text {gen }}^{l} \\
(M W)\end{array}$ & $\begin{array}{c}P_{\text {gen }}^{u} \\
(M W)\end{array}$ & $a_{0}$ & $a_{1}$ & $a_{2}$ & $b_{0}$ & $b_{1}$ & $b_{2}$ \\
\hline 1 & 50 & 200 & 0 & 1.8 & 0.0032 & 22.983 & -0.90 & 0.0126 \\
2 & 20 & 80 & 0 & 1.5 & 0.0154 & 25.313 & -0.10 & 0.0200 \\
5 & 15 & 50 & 0 & 0.8 & 0.0552 & 25.505 & -0.01 & 0.0270 \\
8 & 10 & 35 & 0 & 2.8 & 0.0073 & 24.900 & -0.005 & 0.0291 \\
11 & 10 & 30 & 0 & 2.6 & 0.0221 & 24.700 & -0.004 & 0.0290 \\
13 & 12 & 40 & 0 & 2.6 & 0.0221 & 25.300 & -0.0055 & 0.0271 \\
\hline \hline
\end{tabular}

The typical daily load curves can be derived as follows.

Step 1. Obtain the profile of the daily load curve in daily peak load percentage (Fig. 7) from IEEE RTS [35].

Step 2. Combine this normalized daily mean curve and the total original load of the power grid. A daily curve of the total load of the power grid can be derived. 
Step 3. According to the proportion of each of the original bus load (Table IV), the daily load curve of each bus can be acquired.

TABLE IV

PARAMETERS OF LOADS

\begin{tabular}{ccccccccc}
\hline \hline Bus & $P_{l d}$ & $Q_{l d}$ & Bus & $P_{l d}$ & $Q_{l d}$ & Bus & $P_{l d}$ & $Q_{l d}$ \\
No. & $(M W)$ & $($ MVar $)$ & No. & $($ MW $)$ & $($ MVar $)$ & No. & $($ MW $)$ & $($ MVar $)$ \\
\hline 2 & 21.7 & 12.7 & 12 & 11.2 & 7.5 & 20 & 2.2 & 0.7 \\
3 & 2.4 & 1.2 & 14 & 6.2 & 1.6 & 21 & 17.5 & 11.2 \\
4 & 7.6 & 1.6 & 15 & 8.2 & 2.5 & 23 & 3.2 & 1.6 \\
5 & 94.2 & 19 & 16 & 3.5 & 1.8 & 24 & 8.7 & 6.7 \\
7 & 22.8 & 10.9 & 17 & 9 & 5.8 & 26 & 3.5 & 2.3 \\
8 & 30 & 30 & 18 & 3.2 & 0.9 & 29 & 2.4 & 0.9 \\
10 & 5.8 & 2 & 19 & 9.5 & 3.4 & 30 & 10.6 & 1.9 \\
\hline \hline
\end{tabular}

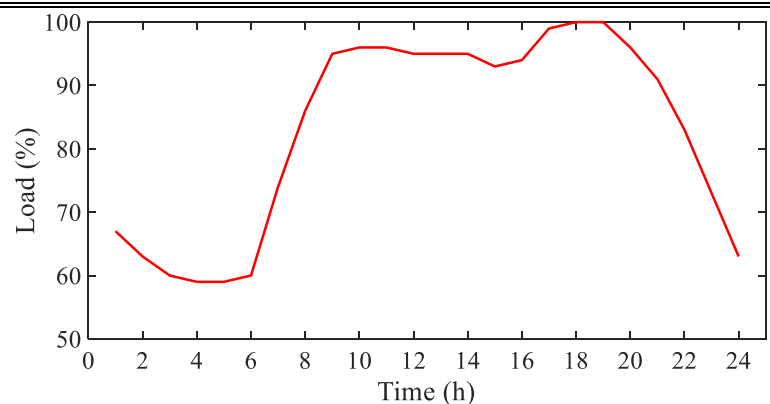

Fig. 7. Daily load curve profile in percent of daily peak (winter, weekday).

The real wind characteristics of Horns Rev I WF measured at $z_{\text {ref }}=62 \mathrm{~m}$ are used. The JPDF of wind speed and direction is shown in Fig. 8. $0^{\circ}$ is the direction of north, $90^{\circ}$ is the direction of east, $180^{\circ}$ is the direction of south and $270^{\circ}$ is the direction of west.

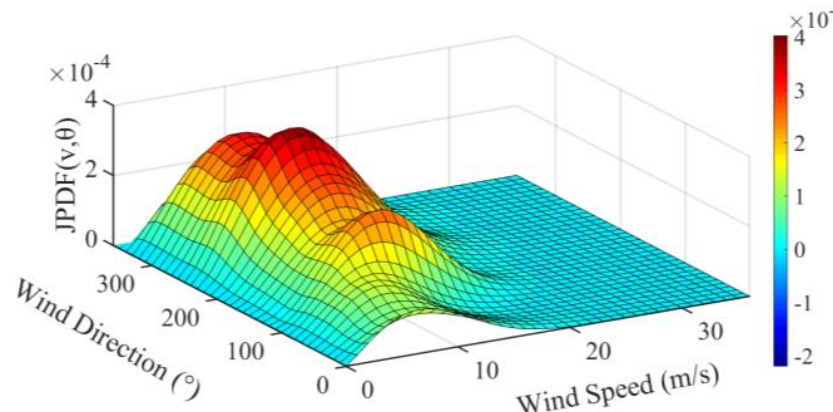

Fig. 8. JPDF of wind speed and direction (01/June/1999-31/May/2002) [36].

The typical daily curves of wind speed and direction are given in Fig. 9, also measured at Horns Rev I WF.

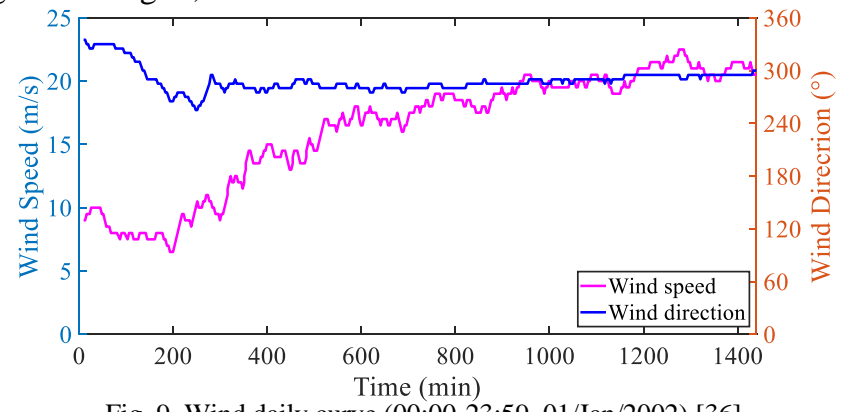

Fig. 9. Wind daily curve (00:00-23:59, 01/Jan/2002) [36].

The parameters of the WGA are set as $N_{w g}=120, N_{g}=10$, $w=0.7298, R=1.5931, m=0.85$, and $I t_{\max }=250$.

\section{B. Simulation Results}

\section{1) Comparison of Different Planning Models}

The bi-hierarchy and the uni-hierarchy planning models are investigated in the WF optimization problem.
Model 1. The proposed bi-hierarchy optimization model using (8) and (19) is applied to determine the WF's optimal capacity and configuration in consideration of the power grid planning and operation costs.

Model 2. The uni-hierarchy optimization model is established by only taking the WTs investment cost in (8) into account, while the operation costs and pollutant emission penalty cost are not included and only Cstr.1 in (8) is regarded. The capacity of the WF is preset as $160 \mathrm{MW}$.

The simulation results are shown in Table V.

$$
\text { TABLE V }
$$

Simulation Results OF DifFERENT PlanNing Models

\begin{tabular}{ccccc}
\hline Model & $\begin{array}{c}F_{1} \\
(€ / M W h)\end{array}$ & $\begin{array}{c}C_{w t} / E_{w f} \\
(€ / M W h)\end{array}$ & $\begin{array}{c}\left(C_{0}+C_{e}\right) / E_{w f} \\
(€ / M W h)\end{array}$ & $C_{w f}(M W)$ \\
\hline Model 1 & 127.78 & 87.65 & 45.92 & 134 \\
Model 2 & 140.03 & 84.23 & 55.80 & 160 \\
\hline
\end{tabular}

It can be indicated from Table $\mathrm{V}$, that in Model 1, the cost of system operation and pollutant emission penalty per unit WF output power takes up a considerable part of the total cost (around 34\%). Therefore, $C_{o}$ and $C_{e}$ should not be omitted and the bi-hierarchy optimization is necessary for WF planning.

The integration of the WF in the electrical network relieves the power generation pressure of other traditional generators which emit considerable amount of pollutant gas. Wind energy, which is a substitute of traditional generation source, makes a great contribution to alleviating the air pollution problem and helps save remarkable expenditure on pollutant penalty cost.

\section{2) Comparison of SPL-constrained and Unconstrained Cases}

To investigate the impact of the inclusion of noise emission of the WF in the optimization problem, the cases with and without the SPL constraint are studied respectively. The simulation results are given in Fig. 10 and Table VI.

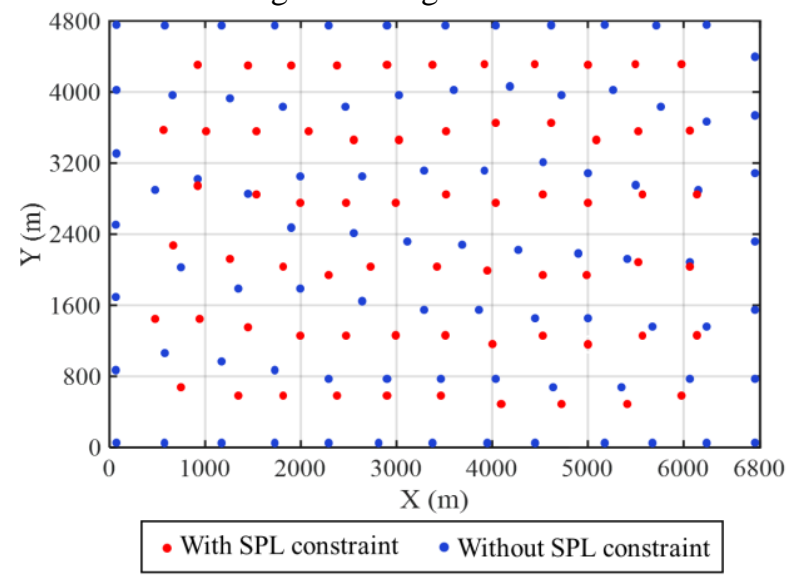

Fig. 10. Comparison of WF optimization with and without $S P L$ constraint. TABLE VI

SIMULATION RESULTS OF CASES WITH AND WITHOUT SPL CONSTRAINT

\begin{tabular}{cccc}
\hline \hline Case & $\begin{array}{c}F_{1} \\
(€ / M W h)\end{array}$ & $\begin{array}{c}\text { Total power } \\
(\mathrm{MW})\end{array}$ & $\begin{array}{c}\text { Average } S P L \\
(\mathrm{~dB}(\mathrm{~A}))\end{array}$ \\
\hline With $S P L$ constraint & 127.78 & 61.32 & 37.52 \\
Without $S P L$ constraint & 116.01 & 87.63 & 46.13 \\
\hline \hline
\end{tabular}

It can be inferred from Fig. 10 that the total number of the WTs installed without the $S P L$ constraint $\left(N_{t}=86\right)$ is more than that with the $S P L$ constraint $\left(N_{t}=67\right)$. There are many WTs installed on the boundaries of the WF when the $S P L$ constraint is absent. This can be explained as follows: when there is no restriction of WF noise emittance, the aim of this optimization will simply become to produce utmost power and 
to minimize the expenditure. When the $S P L$ constraint is added, the value of the objective function $F_{1}$ increases $10.1 \%$ but the average $S P L$ decreases $18.6 \%$.

The $S P L$ received by the receptors No.1-8 in Fig. 5 are shown in Fig. 11. Significant $S P L$ reductions are observed when the $S P L$ constraint is included and only in this case, the $S P L$ limit $\left(S P L_{\text {lim }}=40 d B(A)\right)$ can be satisfied by all the receptors. Therefore, the $S P L$ constraint must be added in the WF planning to ensure all the residents living nearby are not affected by the noise produced by the WF.

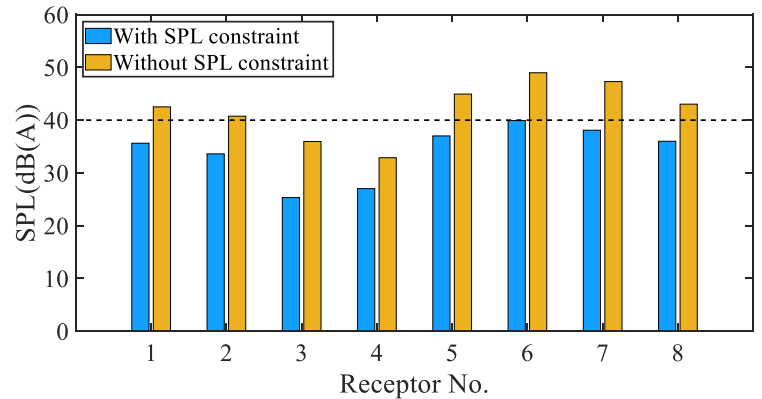

Fig. 11. $S P L$ received by the receptors with and without $S P L$ constraint.

3) Comparison of the WGA and Other Optimization Algorithms

The WGA is compared with the GA and PSO algorithm in the WF optimization problem (case: Model 2). The codes of the algorithms are obtained from [37] and the simulations are carried out in Matlab 2018b, on a PC with an Intel(R) DualCore at $2.40 \mathrm{GHz}$ processor and $8.0 \mathrm{~GB}$ of RAM. The parameters of the GA and PSO algorithms are set as in Table VII. Because the performances of EAs can vary greatly depending on initial settings, the population sizes $(n P o P)$ and the maximum number of iterations $\left(I t_{\max }\right)$ have the same values for each algorithm. Besides, the value of the personal learning coefficient $c_{1}$ in PSO is equal to $R$ in (28). $p_{c}$ and $p_{m}$ are the cross-over and mutation rates in the GA respectively. $c_{2}$ is the global learning coefficient in the PSO.

Table VIII shows the optimization results of $F_{1}$ and runtime (RT) for the 10 trails. Fig. 12 compares the best results of each algorithm in the 10 times of simulations. It can be inferred that the WGA is the best optimization algorithm regarding the ability of searching for the optimal solution $\left(F_{1}=154.86,145.33\right.$ and $140.03 € / M W h$ for the GA, PSO and WGA respectively) as well as the convergence speed (220, 174 and 83 generations for the GA, PSO and WGA respectively). It is mainly because the WGA absorbs the advantages of many other optimization algorithms, such as the GA and PSO algorithm. In this way, the WGA has a better ability of randomly searching in the solution space of the objective function, independent of the features of the function.

TABLE VII

PARAMETERS OF THE GA AND THE PSO ALGORITHM

\begin{tabular}{cccccc}
\hline \hline Parameter & $n P o P$ & $I t_{\max }$ & $p_{c}$ & $p_{m}$ & \\
\hline GA & 120 & 250 & 0.7 & 0.3 & \\
Parameter & $n P o P$ & $I t_{\max }$ & $w$ & $c_{1}$ & $c_{2}$ \\
PSO & 120 & 250 & 0.7298 & 1.5931 & 2.0 \\
\hline \hline
\end{tabular}

TABLE VIII

TEN TIMES RESUlts OF RUNNING THE GA, PSO AND WGA

\begin{tabular}{ccccccc}
\hline \hline Algorithm & \multicolumn{2}{c}{ GA } & \multicolumn{2}{c}{ PSO } & \multicolumn{2}{c}{ WGA } \\
Results & $F_{1}$ & $R T$ & $F_{1}$ & $R T$ & $F_{1}$ & $R T$ \\
& $(€ / M W h)$ & $(s)$ & $(€ / M W h)$ & $(s)$ & $(€ / M W h)$ & $(s)$ \\
\hline 1 & 156.01 & 1684 & 145.64 & 1578 & 141.70 & 1046 \\
2 & 154.92 & 1593 & 145.87 & 1504 & 141.34 & 1109
\end{tabular}

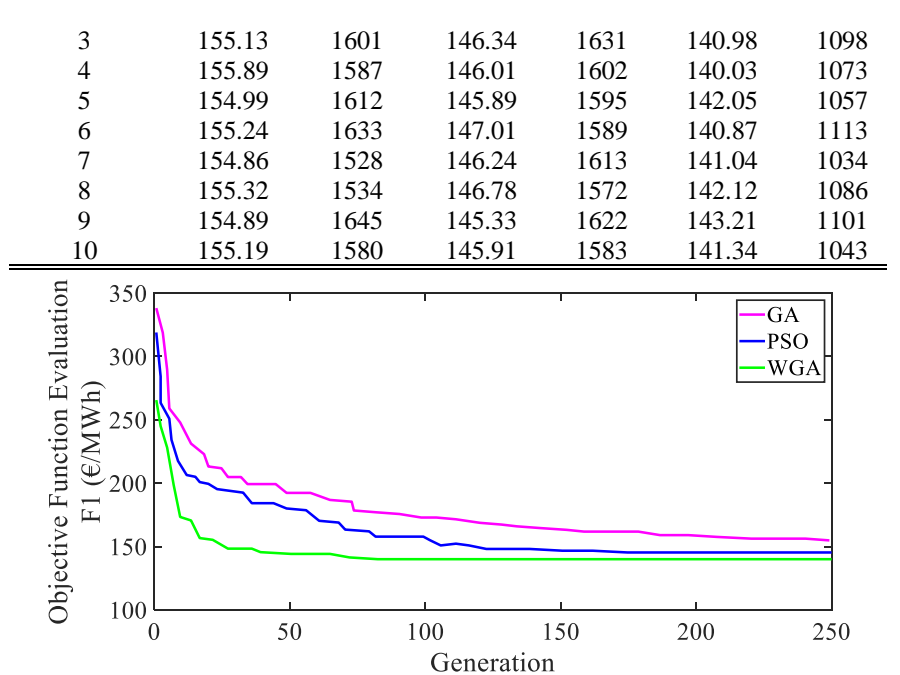

Fig. 12. Convergence characteristics of the GA, PSO and WGA.

\section{CONCLUSION}

The motivation of this paper is to propose a bi-hierarchy optimization framework for a grid-connected WF. The following conclusions can be drawn from the simulation results.

1) The penalty cost of pollutant emissions and the generation cost of the power grid are crucial in the sizing and configuration of a WF. Neglecting these costs will undervalue the profit induced by the connection of the WF to the power grid and the potential of wind energy will be inaccurately estimated. On this account, the bi-hierarchy optimization is more apt than the unihierarchy optimization for the grid-connected WF. However, in reality, WF developers will be separate entities from the grid owners or operators and will not be imposed with pollution penalties outside of their project. In view of this, overall cost optimization is possible where a single entity has control of all generating assets. Where separate owners exist, it is difficult to see the value in such a holistic optimization approach.

2) Overall, enforcing the $S P L$ constraint has a slight effect on the cost of electricity but produces a substantial effect in reducing the noise disturbance to residents in the vicinity of the WF. Hence, it is rewarding to sacrifice a relatively small amount of economic benefit for significant improvements in eliminating the noise annoyance of the WTs.

3) The WGA is an effective EA to solve the WF optimization problem. It can search for the optimum solution with high efficiency and fast convergence speed.

The main contribution and novelty of this paper consists of planning the WF layout by considering the interaction with the electrical power system taking into account the fact that the capacity of the WF is not pre-determined as is the usual practice. It is optimized during the process instead. Also, both the positive and negative environmental impacts brought by the WF are taken into consideration. The extension of this optimization framework to onshore WFs and the inclusion of the WF cable layout in the second layer optimization model can be considered as future work. 


\section{REFERENCES}

[1] 'Wind Power Capacity Worldwide Reaches 600 GW, 53,9 GW added in 2018'. [Online]. Available: http://www/wwindea.org/2018-statistics/.

[2] F. Blaabjerg, and K. Ma, "Wind energy systems," Proc. IEEE, vol. 105, no. 11, pp. 2116-2131, 2017.

[3] G. Mosetti, C. Poloni, and B. Diviacco, "Optimization of wind turbine positioning in large wind farms by means of a genetic algorithm," J. Wind Eng. Ind. Aerodyn., vol. 51, pp. 105-116, 1994.

[4] S. Grady, M. Hussaini, and M. Abdullah, "Placement of wind turbines using genetic algorithms," Renew. Energy, vol. 30, pp. 259-270, 2005.

[5] H. Long and Z. Zhang, "A two-echelon wind farm layout planning model," IEEE Trans. Sustain. Energy, vol. 6, no. 3, pp. 863-871, 2015.

[6] P. Hou, W. Hu, M. Soltani, and Z. Chen, "Optimized placement of wind turbines in large-scale offshore wind farm using particle swarm optimization algorithm," IEEE Trans. Sustain. Energy, vol. 6, no. 4, pp. 1272-1282, 2015.

[7] P. Hou, W. Hu, M. Soltani, C. Chen, B. Zhang, and Z. Chen, "Offshore wind farm layout design considering optimized power dispatch strategy," IEEE Trans. Sustain. Energy, vol. 8, no. 2, pp. 638-647, 2017.

[8] H. Yang, K. Xie, H. Tai, and Y. Chai, "Wind farm layout optimization and its application to power system reliability analysis," IEEE Trans.

Power Syst., vol. 31, no. 3, pp. 2135-2143, 2016.

[9] Y. Wang, H. Liu, H. Long, Z. Zhang, and S. Yang, "Differential evolution with a new encoding mechanism for optimizing wind farm layout," IEEE Trans. Ind. Inform., vol. 14, no. 3, pp. 1040-1054, 2018.

[10] L. Huang, H. Tang, K. Zhang, Y. Fu, and Y. Liu, "3D layout optimization of wind turbines considering fatigue distribution," IEEE Trans. Sustain. Energy, 2018.

[11] S. Sisbot, Ö. Turgut, M. Tunç, and Ü. Çamdalı, "Optimal positioning of wind turbines on Gökceada using multi-objective genetic algorithm," Wind Energy, vol. 13, no. 4, pp. 297-306. 2009.

[12] A. Kusiak and H. Zheng, "Optimization of wind turbine energy and power factor with an evolutionary computation algorithm," Renew. Energy, vol. 35, pp. 685-694, 2010.

[13] J. González, M. Payán, and J. Santos, "A new and efficient method for optimal design of large offshore wind power plants," IEEE Trans. Power Syst., vol. 28, no. 3, pp. 3075-3084, 2013.

[14] L. Chen and E. MacDonald, "A system-level cost-of-energy wind farm layout optimization with landowner modeling," Energy Convers. Manage., vol. 77, pp. 484-494, 2014

[15] S. Shamshirband, D. Petkovic, Z. Cojbašic, V. Nikolic, N. Anuar, N. Shuib, M. Kiah, and S. Akib, "Adaptive neuro-fuzzy optimization of wind farm project net profit," Energy Convers. Manage., vol. 80, pp. 229-237, 2014.

[16] S. Sorkhabi, D. Romero, J. Beck, and C. Amon, "Constrained multiobjective wind farm layout optimization: novel constraint handling approach based on constraint programming," Renew. Energy, vol. 126, pp. 341-353, 2018.

[17] J. Cao, W. Zhu, X. Wu, T. Wang, and H. Xu, "An aero-acoustic noise distribution prediction methodology for offshore wind farms," Energies, vol. 12, no. 18, pp. 1-16, 2019.

[18] L. Vicente and P. Calamai, "Bilevel and multilevel programming: a bibliography review," J. Global Optim., vol. 5, no. 3, pp. 291-306, 1994.

[19] Z. Wang, H. Fan, S. Wang, and G. Wang, "A bilevel programming model for the reactive power optimization," Proc. 7th Int. Power Eng. Conf.,

Singapore, pp. 1-4, 2005.

[20] W. Yao, J. Zhao, F. Wen, Y. Xue, and G. Ledwich, "A hierarchical decomposition approach for coordinated dispatch of plug-in electric vehicles," IEEE Trans. Power Syst., vol. 28, no. 3, pp. 2768-2778, 2013.

[21] S. Wei, L. Zhang, Y. Xu, Y. Fu, and F. Li, "Hierarchical optimization for the double-sided ring structure of the collector system planning of large offshore wind farms," IEEE Trans. Sustain. Energy, vol. 8, no. 3, pp. 1029$1039,2017$.

[22] Y. Dai, Z. Ren, K. Wang, W. Li, Z. Li, and W. Yan, "Optimal sizing and arrangement of tidal current farm," IEEE Trans. Sustain. Energy, vol. 9, no. 1, pp. 168-177, 2018.

[23] Z. Ren, Y. Wang, H. Li, X. Liu, Y. Wen, and W. Li, "A coordinated planning method for micrositing of tidal current turbines and collector system optimization in tidal current farms," IEEE Trans. Power Syst., vol. 34, no. 1, pp. 292-302, 2019.

[24] D. Villanueva, and A. Feijóo, "Reformulation of parameters of the logistic function applied to power curves of wind turbines," Electric Power Systems Research, vol. 28, no. 3, 2016, pp. 51-58.

[25] S. Tao, S. Kuenzel, Q. Xu, and Z. Chen, "Optimal micro-siting of wind turbines in an offshore wind farm using Frandsen-Gaussian wake model," IEEE Trans. Power Syst., vol. 34, no. 6, 2019, pp. 4944-4954.
[26] L. Parada, C. Herrera, P. Flores, and V. Parada, "Wind farm layout optimization using a Gaussian-based wake model," Renew. Energy, vol. 107, pp. 531-541, 2017.

[27] A. Feijóo, "On PQ models for asynchronous wind turbines," IEEE Trans. Power Syst., vol. 24, no. 4, pp. 770-778, 2009.

[28] A. Feijóo, and D. Villanueva, "A PQ model for asynchronous machines based on rotor voltage calculation," IEEE Trans. Energy Conv., vol. 31, no. 2, pp. 813-814, 2016.

[29] (2015) Noise: Data and statistics. World Health Organization. [Online]. Available: http://www.euro.who.int/en/health-topics/environment-and health/noise/data-and-statistics.

[30] ISO 9613-2:1996, Acoustics-Attenuation of sound during propagation outdoors-Part 2: General method of calculation (ISO 9613-2:1996), ISO, Geneva, Switzerland, 1996.

[31] P. Venkatesh, R. Gnanadass, and N. Padhy, "Comparison and application of evolutionary programming techniques to combined economic emission dispatch with line flow constraints," IEEE Trans. Power Syst., vol. 18, no. 2, pp. 688-697, 2003.

[32] A. Shefaei and B. Mohammadi-ivatloo, "Wild goats algorithm: an evolutionary algorithm to solve the real-world optimization problems," IEEE Trans. Ind. Inform., vol. 14, no. 7, pp. 2951-2961, 2018.

[33] Z. Tian, W. Wu, and B. Zhang, "A mixed integer quadratic programming model for topology identification in distribution network," IEEE Trans. Power Syst., vol. 31, no. 1, pp. 688-697, 2016.

[34] 'Vestas V80-2.0 Datasheet'. [Online]. Available: https://en.wind-turbinemodels.com/turbines/19-vestas-v80-2.0.

[35] Probability Methods Subcommittee, "IEEE reliability test system," IEEE Trans. Power Syst., vol. PAS-98, no. 6, pp. 2047-2054, 1979.

[36] J. Feng and W. Shen, "Modelling wind for wind farm layout optimization using joint distribution of wind speed and wind direction," Energies, vol. 8, no. 4, pp. 3075-3092, 2015.

[37] 'Binary and Real-Coded Genetic Algorithms in MATLAB', 'Particle Swarm Optimization in MATLAB'. [Online]. Available: https://yarpiz.com/.

Siyu Tao (GS'18) received the B.Sc degree in electrical engineering from Zhejiang University, China, in 2013 and the M.Sc degree in control systems from Imperial College London, U.K., in 2014. She is currently pursuing the $\mathrm{Ph} . \mathrm{D}$ degree at the Department of Electrical Engineering, Southeast University, China. Her research interests include wind energy and power systems.

Qingshan Xu (M'09) received the Ph.D degree in electrical engineering from Southeast University, China, in 2006. He is currently a professor with the Department of Electrical Engineering, Southeast University, China. His research interests include renewable energy and power system control.

Andrés Feijóo is with the Universidade de Vigo, Spain, where he received his $\mathrm{PhD}$ degree in electrical engineering in 1998.

Peng Hou (M'17) received the Ph.D degree in wind power technology from Aalborg University, Denmark, in 2017. He is currently a Senior Electrical System Engineer at SEWPG European Innovation Center.

Gang Zheng is with the College of Civil Aviation, Nanjing University of Aeronautics and Astronautics. 